PROCEEDINGS OF THE

AMERICAN MATHEMATICAL SOCIETY

Volume 30, No. 1, September 1971

\title{
MAPPINGS OF INDECOMPOSABLE CONTINUA
}

\author{
DAVID P. BELLAMY 1
}

\begin{abstract}
Aвstract. We show that every compact metric continuum is a continuous image of some indecomposable compact metric continuum.
\end{abstract}

Herein a continuum denotes a compact connected metric space. J. W. Rogers, Jr. has raised the questions: 'Which continua are continuous images of indecomposable continua?' and 'Is there an indecomposable continuum of which every indecomposable continuum is a continuous image?' [4]. We answer these questions.

Lemma 1. Every continuum irreducible between two points is a continuous image of some indecomposable continuum.

Proof. Let $M$ be a continuum irreducible between two points, $a$ and $b$. Let $D$ denote the well-known indecomposable continuum which is the union of all semicircles in the closed upper half-plane with center $(1 / 2,0)$ and with both endpoints in the Cantor ternary set on the $x$-axis, and all semicircles in the lower half-plane with center

$$
\left((1 / 2)\left((1 / 3)^{n}+(2 / 3)(1 / 3)^{n}\right), 0\right)
$$

for some nonnegative integer $n$ and with both endpoints lying in the Cantor set and on or to the left of the line $x=(1 / 3)^{n}$. (A sketch can be found in $[3$, p. 206], $[2$, p. 332], or $[4$, p. 96], so none is included here.)

Now, let

$$
\begin{gathered}
D_{a}=\{(x, y) \in D: x \leqq 2 / 5\}, \quad D_{b}=\{(x, y) \in D: x \geqq 3 / 5\}, \\
A=\left\{y:(2 / 5, y) \in D_{a}\right\}
\end{gathered}
$$

and observe that also

$$
A=\left\{y:(3 / 5, y) \in D_{b}\right\} .
$$

The continuum $D_{M}$ is now obtained from the disjoint union of $D_{a}, D_{b}$, and $M \times A$ by identifying $(2 / 5, y) \in D_{a}$ with $(a, y) \in M \times A$ and identifying $(3 / 5, y) \in D_{b}$ with $(b, y) \in M \times A$, for each $y \in A$. (Intuitively speaking we have removed from $D$ a copy of a closed interval crossed with $A$ and spliced in a copy of $M \times A$.) The proof that $D_{M}$ is indecomposable is straightforward, and is left to the reader. It de-

Received by the editors December 31, 1970.

AMS 1970 subject classifications. Primary 54F20, 54C05, 54C15.

${ }_{1}$ The author was supported by the University of Delaware Research Foundation. 
pends upon the indecomposability of $D$ and the irreducibility of $M$.

Now, we define $g: D_{M} \rightarrow M$ by

$$
\begin{aligned}
g(p)=a & \text { for } p \in D_{a}, \\
g(p)=b & \text { for } p \in D_{b}, \\
g(m, y)=m & \text { for }(m, y) \in M \times A .
\end{aligned}
$$

This is the desired mapping of $D_{M}$ on to $M$.

TheOREM 2. If $S$ is a continuum, there exists an indecomposable continuum of which $S$ is a continuous image.

Proof. Lemma 2 of [1] states that there is a continuous mapping of some continuum $M$ irreducible between two points onto $S$; and by the above lemma there is a continuous surjection $f: D_{M} \rightarrow M$. The composition of these maps yields a continuous surjection $f: D_{M} \rightarrow S$. Since $D_{M}$ is indecomposable, this is the desired mapping.

COROLlARY 3. There is no continuum of which every indecomposable continuum is a continuous image.

Proof. By the above theorem, every continuum would be a continuous image of such a continuum, contradicting the principal result of $[5]$.

CoRollary 4. If $S$ is a continuum there exists an indecomposable continuum $X$ which contains a copy of $S$ as a retract.

Proof. First, the mapping of $D_{M}$ onto $M$ can be considered a retraction by identifying $M$ with $M \times\{y\}$ for some $y \in A$. In turn, the mapping in Lemma 2 of [1] can be considered a retraction in a similar manner. Since composition of the retractions $g: D_{M} \rightarrow M$ and $r: M \rightarrow S$ yields a retraction, we set $X=D_{M}$ and the proof is complete.

\section{REFERENCES}

1. D. P. Bellamy, A non-metric indecomposable continuum, Duke Math. J. 38 (1971), 15-20.

2. J. G. Hocking and G. S. Young, Topology, Addison-Wesley, Reading Mass., 1961. MR 23 \#A2857.

3. K. Kuratowski, Topologie. Vol. 2, 3rd ed., Monografie Mat., Tom 21, PWN, Warsaw, 1961; English transl., Academic Press, New York; PWN, Warsaw, 1968. MR 24 \#A2958.

4. J. W. Rogers, Jr., Continuous mappings on continua, Proc. Auburn Topology Conference, Auburn University, Auburn, Ala., 1969, pp. 94-97.

5. Z. Waraszkiewicz, Sur un problème de M. H. Hahn, Fund. Math. 22 (1934), 180205.

University of Delaware, Newark, Delaware 19711 